International Journal of Research in Nursing 3 (1): 15-20, 2012

ISSN 1949-0194

C 2012 Science Publications

\title{
Unmet Needs in Chronic Obstructive Pulmonary Disease: A Metasynthesis Protocol
}

\author{
${ }^{1-3}$ Rebecca T. Disler, ${ }^{1-3}$ Anna Green, ${ }^{1-3}$ Tim Luckett, \\ ${ }^{1-3}$ Phillip J. Newton, ${ }^{1-3}$ Sally C. Inglis, ${ }^{3,4}$ David C. Currow and ${ }^{1-3}$ Patricia M. Davidson \\ ${ }^{1}$ Faculty of Health, University of Technology Sydney, \\ New South Wales, Australia P.O. Box 123, Broadway, NSW 2007, Australia \\ ${ }^{2}$ Centre for Cardiovascular and Chronic Care, \\ Faculty of Health, University of Technology Sydney, Australia \\ ${ }^{3}$ Improving Palliative Care through Clinical Trials (Im Pa CCT) NSW, Australia \\ ${ }^{4}$ Flinders Centre for Clinical Change, \\ Faculty of Health Sciences, Flinders University, Adelaide, South Australia, Australia
}

\begin{abstract}
Problem statement: Chronic obstructive pulmonary disease is a chronic progressive illness. Despite the high burden experienced by individuals in the advanced stages of illness, individuals with advanced COPD continue to have unmet needs and limited access to palliative care. This Metasynthesis seeks to describe: the barriers and facilitators care access and provision; the unmet needs of individuals with advanced COPD, their families and carers; and the experiences of health professionals. Data sources: Medline, PsychINFO, AMED, CINAHL and Sociological Abstracts were searched for articles published between 1990 and December 31st 2011. Medical Subject Headings (MeSH) and key words will be used to guide the search. The strategy will be reviewed by the CareSearch palliative knowledge network and a health informatics expert. Approach: Metasyntheses are increasingly used to gain new insights and understandings of complex research questions through the amalgamation of data from individual qualitative studies. The principles of the Preferred Reporting Items for Systematic Reviews and Meta-Analyses (PRISMA) and thematic synthesis will be used to achieve consistent reporting and transparency of methods. Results: Inclusion of studies, quality assessment and allocation of free codes into EPPI-Reviewer 4 software will be carried out by two independent investigators. Auditing of random cases will be undertaken and disagreements resolved through group discussion of an expert panel. Descriptive and analytical themes will be developed through thematic synthesis and expert panel discussion. Conclusion: Qualitative data provide useful information in understanding the individual's unique experience. Combining discrete qualitative studies provides an important opportunity to provide a voice to patients, their families and professional careers in managing advanced COPD.
\end{abstract}

Key words: Medical Subject Headings (MeSH), Preferred Reporting Items for Systematic Reviews and Meta-Analyses (PRISMA), Chronic Obstructive Pulmonary Disease (COPD)

\section{PROBLEM STATEMENT}

Chronic Obstructive Pulmonary Disease (COPD) is a life-limiting illness affecting 80 million people worldwide, is the fourth most prevalent cause of death globally and accounts for $3.5 \%$ of total years lost due to disability (WHO, 2008). Despite the high symptom burden, individuals with advanced COPD continue to experience unmet needs and have limited access to palliative care at the end-of-life (Elkington et al., 2005; Gore et al., 2000; Luddington et al., 2001; Curtis, 2008; Gysels and Higginson, 2008).

Corresponding Author: Rebecca T. Disler, Faculty of Nursing Midwifery and Health, University of Technology Sydney, New South Wales, Australia, P.O. Box 123, Broadway, NSW 2007, Australia
A focus on acute care and challenges in predicting the end-stage of disease creates a milieu that is commonly reactive and ad hoc rather than an approach which is responsive to individuals' ongoing needs. (Elkington et al., 2005; Gore et al., 2000; Luddington et al., 2001; Curtis, 2008; Gysels and Higginson, 2008).

The fluctuating and episodic decline is characteristic of the COPD trajectory and makes it difficult to determine when death is likely (CreaghBrown and Shee, 2008; Curtis, 2008; Gardiner et al., 2009; Hansen-Flaschen, 2004). 'Life-limiting' illness is 
defined as an illness where death will likely be a consequence of the specified illness and likely shorten an individual's life (Clayton et al., 2007). The term 'life limiting' illness is inclusive of non-malignant terminal diseases such as COPD (Steinhauser et al., 2011). While not synonymous with advanced COPD, 'Severe COPD' or 'Stage 3' COPD is defined by the Global Initiative for Chronic Obstructive Lung Disease (GOLD) as 'characterized by further worsening of airflow limitation (FEV1/FVC $<0.7 ; 30 \%<$ FEV1 < $50 \%$ predicted)' along with 'greater shortness of breath, reduced exercise capacity, fatigue and repeated exacerbations that almost always have an impact on patients' quality of life' (GICOLD, 2011). Independent predictors of death from COPD include: hospitalization; functional limitation; severe reduction in lung function; use of long term oxygen therapy; depression; low nutritional state; and the presence of co-morbidities (Gore et al., 2000; Hansen-Flaschen, 2004; Pinnock et al., 2011; Murray et al., 2006). Additionally a clinician's assessment that an individual is unlikely to survive two years is also seen as a useful prognostic tool (Hansen-Flaschen, 2004; Murray et al., 2006).

Metasyntheses have increasingly been used to gather qualitative data from different studies in order to gain an understanding of complex research questions (Thomas and Harden, 2008; Walsh and Downe, 2005). The integration of a number of individual qualitative studies is argued to provide an interpretation that 'will not be found in any one research report but, rather, are inferences derived from taking all of the reports in a sample as a whole' (Sandelowski et al., 1997). Through the amalgamation and syntheses of these data new insights and meanings may be generated which may not have been evident in individual qualitative studies (Sandelowski et al., 1997; Thomas and Harden, 2008; Walsh and Downe, 2005).

In the context of health care, metasyntheses are used to 'enrich understanding of complex and multifaceted health experiences and health care practices' (Walsh and Downe, 2005). The outcomes of metasyntheses are not merely summative of individual qualitative studies but also cast a lens on a broader experiential perspective (Walsh and Downe, 2005).

This technique also responds to a desire to make qualitative data and the unique perspective it presents, accessible to wider audiences who may not have the capacity to interpret large bodies of qualitative evidence (Thomas and Harden, 2008). Metasyntheses consequently have the potential to increase the leverage of qualitative data to inform policy and practice (Thomas and Harden, 2008).
The conduct of metasyntheses has not been without contention (Thomas and Harden, 2008; Walsh and Downe, 2005). Separating qualitative data from the timing and participant characteristics of each study opens authors to accusations of 'de-contextualising findings and wrongly assuming that there are commensurable' (Thomas and Harden, 2008). However the summarizing and synthesis of primary data (i.e., Direct quotations) and themes identified by article authors does allow metasyntheses to summarize the individuals' experience within the context of the study (Thomas and Harden, 2008; Walsh and Downe, 2005).

This metasynthesis will allow the authors to explore the complexities of individuals' experience of advanced COPD (Thomas and Harden, 2008; Walsh and Downe, 2005). Developing greater insight into the experience of COPD-including the unmet needs and barriers faced by individuals with advanced COPD, their families and the healthcare professionals who care for them-has unique potential for informing the coordination and responsiveness of services for this burdensome condition.

In exploring individuals' experiences of illness, we take this to be a constructed and experiential phenomenon concerned with how individuals come to understand and live with their illness and its management (Conrad and Barker, 2010). We anticipate that this will include not only perceptions of how disease and management have impacted daily life (e.g., through symptom burden) but also cultural and societal meaning for the individual as well as the way in which society interacts with individuals with illness (Conrad and Barker, 2010). Exploration of unmet needs will be based on those experiences in which individuals require assistance but for which inadequate management is in place. In the context of chronic illness, individuals' needs may include physical, psychological, cultural, existential, sexual and financial (Desai et al., 2001; Elkington et al., 2005; Gore et al., 2000).

The objectives of this review are to describe:

- The barriers and facilitators to care access and provision for individuals with advanced COPD, their families and carers and health professionals

- The unmet needs of individuals with advanced COPD and their families and

- The experiences of healthcare professionals caring for individuals with advanced COPD

\section{Approach:}

Methods: The principles of the Preferred Reporting Items for Systematic Reviews and Meta-Analyses (PRISMA) approach have been used to achieve 
Intl. J. Res. Nursing 3 (1): 15-20, 2012

consistent reporting and transparency of the methods used in this metasynthesis (Moher et al., 2009).

\section{Eligibility criteria:}

Studies will be included if:

- Reported in English

- Published in a peer reviewed journal between 1990 and December 2011

- The publication reports the perspectives of individuals with advanced COPD and/or their family/carers and/or health professionals involved in COPD management

- The publication contains qualitative data; that is, presentation and or synthesis of primary data (i.e., direct quotations) and themes identified by article authors. To ensure a minimum quality standard, we will require reports to include at least one excerpt of raw data; and

- Data collection included interviews, focus groups, or open-ended survey questions

Studies with samples that include but are not limited to COPD individuals, family/carers and health professionals will be included provided COPD-specific data can be extracted. Qualitative data from mixed methods studies will also be included if the above criteria are met.

Population: For the context of this metasynthesis, individuals with advanced COPD are defined as those with an advanced life-limiting illness and predictors associated with adverse outcomes (Gore et al., 2000; Hansen-Flaschen, 2004; Pinnock et al., 2011; Murray et al., 2006). Family and carers (both informal and formal) will be taken to be any significant person involved in the care and management of individuals with advanced COPD (Seamark et al., 2004; Spence et al., 2008; Gysels and Higginson, 2009). Healthcare professionals will to include healthcare professionals who discuss their experiences of management of advanced COPD, including nurses, physicians and allied health professionals (Blackler et al., 2004; Goodridge et al., 2008).

Information sources: The following electronic databases - Medline, PsychINFO, AMED, CINAHL and Sociological Abstracts-will be searched for articles published between 1990 and February 29th 2012. These dates were decided to reflect contemporaneous approaches to COPD management, particularly approaches to chronic disease management. Included article reference lists will be hand searched for additional studies.
Table 1 Search Strategy (Medline example)

\begin{tabular}{|c|c|}
\hline exp Pulmonary Disease, Chronic Obstructive/ & 17330 \\
\hline COPD*.mp. & 19799 \\
\hline exp Airway Obstruction/ & 18032 \\
\hline airway obstruction.mp. & 21470 \\
\hline $\mathrm{COAD}^{*} \cdot \mathrm{mp}$ & 185 \\
\hline Exp Lung Diseases, Obstructive/ & 144900 \\
\hline 1 or 2 or 3 or 4 or 5 or 6 & 49454 \\
\hline advance care planning.mp. or exp Advance Care Planning/ & 6307 \\
\hline exp Attitude to Death/ or attitude to death.mp. & 12505 \\
\hline bereave\$.mp. or exp Bereavement/ & 10837 \\
\hline Death/ or death.mp. & 467014 \\
\hline dying.mp. & 22516 \\
\hline end of life.mp. & 9102 \\
\hline hospice\$.mp. or exp Hospices/ & 9912 \\
\hline exp Palliative Care/ or palliat\$.mp. & 57391 \\
\hline exp Terminal Care/ or terminal\$.mp. & 392871 \\
\hline 7 or 8 or 9 or 10 or 11 or 12 or 13 or 14 or 15 & 899336 \\
\hline 6 and 16 & 3367 \\
\hline exp Interview/ or interview\$.mp. & 207087 \\
\hline experience.mp. & 378908 \\
\hline exp Qualitative Research/ or qualitative.mp. & 102207 \\
\hline 18 or 19 or 20 & 640536 \\
\hline 17 and 21 & 292 \\
\hline limit 23 to English language & 261 \\
\hline limit 24 to "all adult (19 plus years)" & 182 \\
\hline
\end{tabular}

* COPD: chronic obstructive pulmonary disease, * COAD: chronic obstructive airways disease, \$: wildcard, exp: explode terms

Search: Medical Subject Headings (MeSH) and key words will be used to guide the search including the terms outlined in Table 1 for Medline and will be adjusted as appropriate for each database. These search terms and strategies have been reviewed by the CareSearch palliative knowledge network (CareSearch, 2012). Terms of filtering for qualitative research were those recommended by the McMaster University Health Information Research Unit (HIRU, 2011). The overall search strategy will be reviewed by a health informatics expert (Fig. 1).

Study selection: Inclusion of studies will be independently assessed by two researchers specialized in COPD, palliative and chronic care (RTD, PMD). These entries will be checked by a third independent investigator (AG) through random selection and checking. Disagreements will be resolved by consensus (Fig. 2).

Data management: Data from the selected papers will be entered into EPPI-Reviewer 4 software by one investigator (RTD) to allow for assessment against inclusion criteria and coding by multiple investigators (Thomas and Harden, 2008). These entries will be checked by a second investigator (AG) through random selection and checking. Any disagreements over quality assessment will be resolved through group discussion (Walsh and Downe, 2005).

Descriptive details of the selected studies will be recorded with country of origin, sample characteristics (participant type, setting and demographics), study characteristics (method, conceptual approach) and results (themes identified by the authors). Where 
possible description of the stage of illness trajectory will be collected, as well data on family and career relationship to the individual and the characteristics of the assistance they provide. Provider data will be collected on discipline and role in patient care.

Quality assessment: The quality of studies will be independently evaluated with by two investigators (RTD, AG) with the checklist developed by Kitto et al. (2008). This checklist evaluates studies in terms of clarity of aims/research question, justification of the approach used, procedural rigor, representativeness of the sample, interpretation of the data, reflexivity and evaluative rigor and transferability of findings (Kitto et al., 2008; Walsh and Downe, 2005).

Synthesis of results (Fig. 2): This metasynthesis will use a thematic synthesis of qualitative research as developed by (Thomas and Harden, 2008; Barnett-Page and Thomas, 2009). It is anticipated that the systematic approach to combining qualitative data in this metasynthesis will generate new insights and meanings which may not have been evident in the discrete studies (Sandelowski et al., 1997; Thomas and Harden, 2008; Walsh and Downe, 2005).

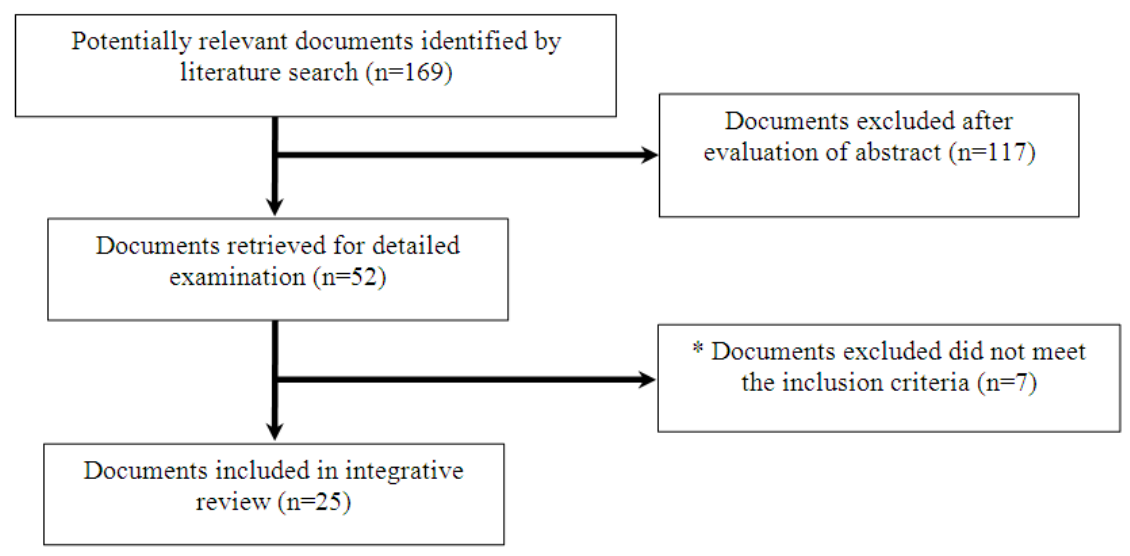

Fig. 1: Flow chart of studies from search for inclusion (Medline example)

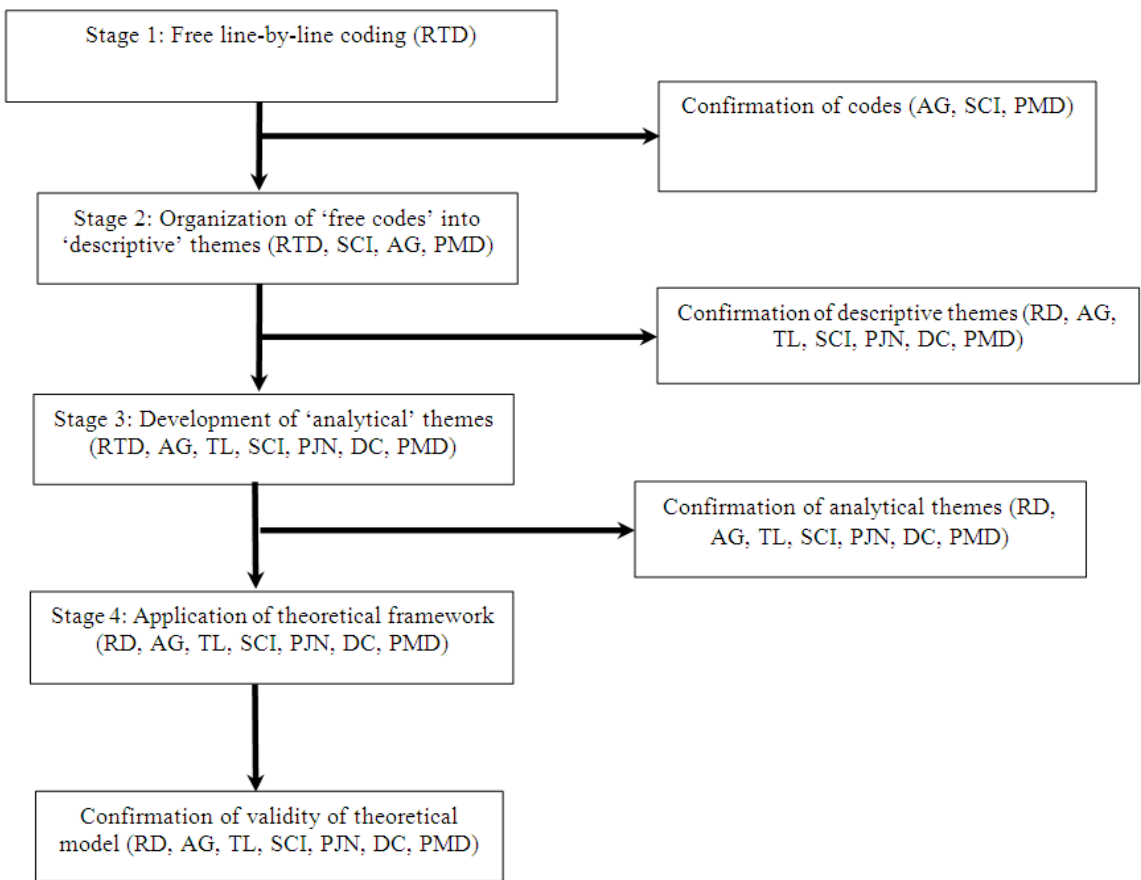

Fig. 2: Flow chart of data synthesis 
Stage 1: Free line-by-line coding: Line-by-line free coding will be carried out by two independent investigators (RTD, AG). Free codes will be kept as close to the primary data as possible (Thomas and Harden, 2008). Coding will be carried out on the presentation and/or synthesis of primary data (interpreted within the context of the setting) of the perspectives of individuals with advanced COPD, or their family and careers, or health professionals (Thomas and Harden, 2008). The trustworthiness of the data will be ensured through random selection and checking (PMD, SCI). Disagreements will be resolved by consensus of four reviewers (RTD, PMD, AG, TL).

Stage 2: organization of 'free codes' into related areas to construct 'descriptive' themes: The free codes will be organized into descriptive themes by three investigators initially (RTD, AG, PMD) and then confirmed through discussion of an expert panel in COPD, palliative and chronic care (RTD, AG, TL, SCI, PJN, DC, PMD) (Walsh and Downe, 2005; Thomas and Harden, 2008). In the first instance, codes will be grouped into a topic area and super- and sub-ordinate relationships. Disagreements in descriptive themes will be resolved through group discussion (Walsh and Downe, 2005; Thomas and Harden, 2008).

Stage 3: Development of 'analytical' themes: An expert panel in COPD, palliative and chronic care will be used to develop central themes and analytical inferences not overtly evident in the initial codes (RTD, AG, TL, SCI, PJN, DC, PMD) (Thomas and Harden, 2008; Walsh and Downe, 2005). The development of analytical themes will allow for a broader understanding and interconnectedness between themes within the data (Walsh and Downe, 2005; Thomas and Harden, 2008). A theoretical framework will be sought to assist in the organization of analytical themes organizing the relationships between those issues found to be central to individual with advanced COPD, their family and carers and health professionals (Lovell, 2012).

Rigor: The use of qualitative primary data is an inductive, reflective and critical process in theme development and understanding of complex issues (Walsh and Downe, 2005). Rigor is supported in qualitative research through reflexivity and systematic approaches to data analysis, interpretation and synthesis (Barnett-Page and Thomas, 2009). The transparent presentation of the method of undertaking metasynthesis, as well as the use of independent investigators and panel discussion to promote the validity of findings increases the rigor of this metasythnesis (Thomas and Harden, 2008).

\section{CONCLUSION}

Qualitative data provide useful information in understanding the individual's unique experience. Combining discrete qualitative studies provides an important opportunity to provide a voice to patients, their families and professional careers in managing advanced COPD.

\section{ACKNOWLEDGEMENT}

No financial support was provided and no conflicts of interest exist within this research.

Authors' contributions: Conception and design RTD, SCI, PMD; acquisition of data RTD; analysis and interpretation of data RTD, AG, TL, SCI, PJN, DC, PMD; drafting the manuscript RTD, AG, TL, SCI, PJN, DC, PMD. All authors have given final approval of the version to be submitted.

\section{REFERENCES}

Barnett-Page, E. and J. Thomas, 2009. Methods for the synthesis of qualitative research: A critical review. BMC Med. Res. Methodol, 9: 59-59. DOI: 10.1186/1471-2288-9-59

Blackler, L., C. Mooney and C. Jones, 2004. Palliative care in the management of chronic obstructive pulmonary disease. Br. J. Nurs., 13: 518-521. PMID: 15215727

CareSearch, 2012. Palliative Care PubMed Searches. CareSearch Palliative Care Knowledge Network.

Clayton, J.M., K.M. Hancock, P.N. Butow, M.H.N. Tattersall and D.C. Currow et al., 2007. Clinical practice guidelines for communicating prognosis and end-of-life issues with adults in the advanced stages of a life-limiting illness and their caregivers. Med. J. Aust., 186: S77-S108. PMID: 17727340

Conrad, P. and K.K. Barker, 2010. The social construction of illness: Key insights and policy implications. J. Health Soc. Behav., 51: S67-S79. DOI: $10.1177 / 0022146510383495$

Creagh-Brown, B.C. and C.D. Shee, 2008. Noninvasive ventilation as ceiling of therapy in end-stage chronic obstructive pulmonary disease. Chronic Respiratory Dis., 5: 143-148. DOI: $10.1177 / 1479972308089234$

Curtis, J.R., 2008. Palliative and end-of-life care for patients with severe COPD. Eur. Respir. J., 32: 796-803. PMID: 17989116

Desai, M.M., H.R. Lentzner and J.D. Weeks, 2001. Unmet need for personal assistance with activities of daily living among older adults. Gerontologist, 41: 82-88. PMID: 11220818 
Elkington, H., P. White, J. Addington-Hall, R. Higgs and P. Edmonds, 2005. The healthcare needs of chronic obstructive pulmonary disease patients in the last year of life. Palliative Med., 19: 485-491. DOI: 10.1191/0269216305pm1056oa

Gardiner, C., M. Gott, N. Small, S. Payne and D. Seamark et al., 2009. Living with advanced chronic obstructive pulmonary disease: Patients concerns regarding death and dying. Palliative Med., 23: 691-697. DOI: $10.1177 / 0269216309107003$

GICOLD, 2011. Global Strategy for the Diagnosis, Management and Prevention of Chronic Obstructive Pulmonary Disease. Global Initiative for Chronic Obstructive Lung Disease Inc.

Goodridge, D., W. Duggleby, J. Gjevre and D. Rennie, 2008. Caring for critically ill patients with advanced COPD at the end of life: A qualitative study. Intensive Crit. Care Nurs., 24: 162-170. PMID: 18313923

Gore, J.M., C.J. Brophy and M.A. Greenstone, 2000. How well do we care for patients with end stage Chronic Obstructive Pulmonary Disease (COPD)? A comparison of palliative care and quality of life in COPD and lung cancer. Thorax Int. J. Respiratory Med., 55: 1000-1006. DOI: 10.1136/thorax.55.12.1000

Gysels, M. and I.J. Higginson, 2008. Access to services for patients with chronic obstructive pulmonary disease: The invisibility of breathlessness. J. Pain Symptom Manage., 36: 451-460. PMID: 18495412

Gysels, M.H. and I.J. Higginson, 2009. Caring for a person in advanced illness and suffering from breathlessness at home: Threats and resources. Pall. Supp. Care, 7: 153-162. PMID: 19538797

Hansen-Flaschen, J., 2004. Chronic obstructive pulmonary disease: the last year of life. Respiratory Care, 49: 90-98.

Kitto, S., J. Chesters and C. Grbich, 2008. Quality in qualitative research: Criteria for authors and assessors in the submission and assessment of qualitative research articles for the Medical Journal of Australia. Med. J. Aus., 188: 243-246.

Lovell, M., 2012. Assessment and management of adult cancer pain: lessons from a systematic review and synthesis of qualitative studies. Anaesthisiology Intensive Care, 40: 543-544.

Luddington, L., S. Cox, I. Higginson and B. Livesley, 2001. The need for palliative care for patients with non-cancer diseases: A review of the evidence. Int. J. Palliat. Nurs., 7: 221-226. PMID: 12148972
HIRU, 2011. Search Filters for MEDLINE in Ovid Syntax and the PubMed translation. Health Information Research Unit.

Moher, D., A. Liberati, J. Tetzlaff and D.G. Altman, 2009. Preferred reporting items for systematic reviews and meta-analyses: The Prisma statement. Ann. Intern. Med., 151: 264-269. PMID: 19622511

Murray, S.A., H. Pinnock and A. Sheikh, 2006. Palliative care for people with COPD: We need to meet the challenge. Primary Care Respiratory J., 15: 362-364. DOI: 10.1016/j.pcrj.2006.08.008

Pinnock, H., M. Kendall, S.A. Murray, A. Worth and P. Levack et al., 2011. Living and dying with severe chronic obstructive pulmonary disease: Multiperspective longitudinal qualitative study. BMJ, 342: d142- d142. DOI: 10.1136/bmj.d142

Sandelowski, M., S. Docherty and C. Emden, 1997. Qualitative metasynthesis: Issues and techniques. Res. Nursing Health, 20: 356-371. DOI: 10.1002/(SICI)1098240X(199708)20:4<365::AID-NUR9>3.0.CO;2-

Seamark, D.A., S.D. Blake, C.J. Seamark and D.M. Halpin, 2004. Living with severe Chronic Obstructive Pulmonary Disease (COPD): Perceptions of patients and their carers. An interpretative phenomenological analysis. Pall. Med., 18: 619-625. PMID: 15540670

Spence, A., F. Hasson, M. Waldron, G. Kernohan and D. McLaughlin et al., 2008. Active carers: Living with chronic obstructive pulmonary disease. Int. J. Palliat. Nurs., 14: 368-372. PMID: 19023952

Steinhauser, K.E., R.M. Arnold, M.K. Olsen, J. Lindquist and J. Hays et al., 2011. Comparing three life-limiting diseases: Does diagnosis matter or is sick, sick. J. Pain Symptom Manage., 42: 331341. PMID: 21276704

Thomas, J. and A. Harden, 2008. Methods for the thematic synthesis of qualitative research in systematic reviews. BMC Med. Res. Methodol.

Walsh, D. and S. Downe, 2005. Meta-synthesis method for qualitative research: A literature review. J. Adv. Nurs., 50: 204-211. DOI: 10.1111/j.13652648.2005.03380. $\mathrm{x}$

WHO, 2008. The Global Burden of Disease: 2004 Update. 1st Edn., World Health Organization, Geneva, ISBN-10: 9241563710, pp: 146. 